\title{
Microphotonic Forces from Superfluid Flow
}

\author{
D. L. McAuslan, G. I. Harris, C. Baker, ${ }^{*}$ Y. Sachkou, X. He, E. Sheridan, and W. P. Bowen \\ Centre for Engineered Quantum Systems, School of Mathematics and Physics, \\ University of Queensland, Brisbane, Queensland 4072, Australia
}

(Received 23 December 2015; published 29 April 2016)

\begin{abstract}
In cavity optomechanics, radiation pressure and photothermal forces are widely utilized to cool and control micromechanical motion, with applications ranging from precision sensing and quantum information to fundamental science. Here, we realize an alternative approach to optical forcing based on superfluid flow and evaporation in response to optical heating. We demonstrate optical forcing of the motion of a cryogenic microtoroidal resonator at a level of $1.46 \mathrm{nN}$, roughly 1 order of magnitude larger than the radiation pressure force. We use this force to feedback cool the motion of a microtoroid mechanical mode to $137 \mathrm{mK}$. The photoconvective forces we demonstrate here provide a new tool for high bandwidth control of mechanical motion in cryogenic conditions, while the ability to apply forces remotely, combined with the persistence of flow in superfluids, offers the prospect for new applications.
\end{abstract}

DOI: 10.1103/PhysRevX.6.021012

Optical forces are widely utilized in photonic circuits $[1,2]$, micromanipulation $[3,4]$, and biophysics $[5,6]$. In cavity optomechanics, in particular, optical forces enable cooling and control of microscale mechanical oscillators that can be used for ultrasensitive detection of forces, fields and mass [7-9], quantum and classical information systems [10], and fundamental science [11,12]. Recent progress has seen radiation pressure used for coherent state swapping [13], ponderomotive squeezing [14], and ground state cooling [15], while static gradient forces have enabled all-optical routing [16] and nonvolatile mechanical memories [17]. Likewise, photothermal forces, where the mechanical element moves in response to mechanical stress from localized optical absorption and heating, have been used to demonstrate cavity cooling of a semiconductor membrane [18,19], single molecule force spectroscopy [20], and rich chaotic dynamics in suspended mirrors [21].

Here, we demonstrate an alternative photoconvective approach to optical forcing that allows an order-ofmagnitude stronger mechanical actuation than radiation pressure. In our implementation, this technique utilizes the convection in superfluids, whereby frictionless fluid flow is generated in response to a local heat source. This well-known superfluid fountain effect [22] is a direct manifestation of the phenomenological two-fluid model proposed by Landau [23] and Tisza [24]. The momentum carried by the helium-4 flow is then transferred to a mechanical element via collision

\footnotetext{
* Corresponding author. c.baker3@uq.edu.au

Published by the American Physical Society under the terms of the Creative Commons Attribution 3.0 License. Further distribution of this work must maintain attribution to the author $(s)$ and the published article's title, journal citation, and DOI.
}

Subject Areas: Photonics, Quantum Physics, Superfluidity

and recoil of superfluid atoms. If the heat source is localized upon the mechanical element, the incident superfluid atoms are either converted to a normal fluid counterflow or evaporated [see Figs. 1(a) and 1(b)]. Alternatively, a distant heat source could be utilized with the mechanical element acting to reroute the superflow.

Strong actuation forces are important for a range of techniques in quantum optomechanics, and, most particularly, for protocols that utilize precise measurements combined with feedback actuation to generate nonclassical states of a mechanical oscillator. With linear measurements, such protocols, for instance, allow ground state cooling [25] and squeezed state generation [26-28], while with nonlinear measurements, they are predicted to enable the generation of phonon number states [29] and mechanical superposition states [30]. Currently, of all optical techniques, photothermal forces provide the strongest actuation capabilities at room temperature [31]. However, due to the reduced thermal expansion of materials at low temperatures, they are ineffective in the cryogenic conditions typical for quantum optomechanics experiments. The superfluid photoconvective forces we demonstrate here provide an alternative pathway to achieve strong optical actuation in cryogenic conditions. Furthermore, due to the presence of superflow, superfluid helium-4 has the largest thermal conductivity of any known material. This could be used to greatly reduce localized heating of the mechanical element, which often constrains the performance of cryogenic quantum optomechanics experiments [32]. More broadly, since the superfluid flow can be generated in a location remote from the mechanical element, superfluid photoconvective forces offer the prospects for remote actuation, a capability that is not available with any cryogenic actuation technique and could be used, for instance, to apply torques at microscale 
(a)

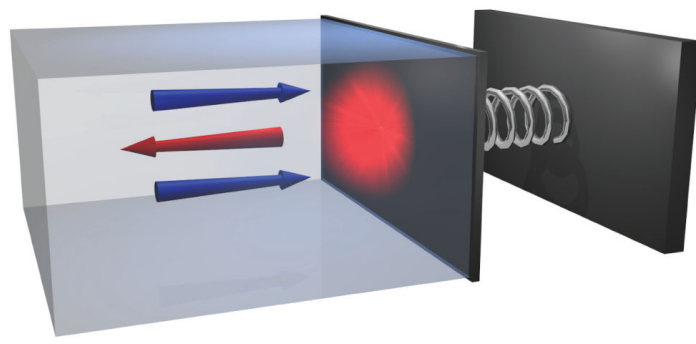

(b)

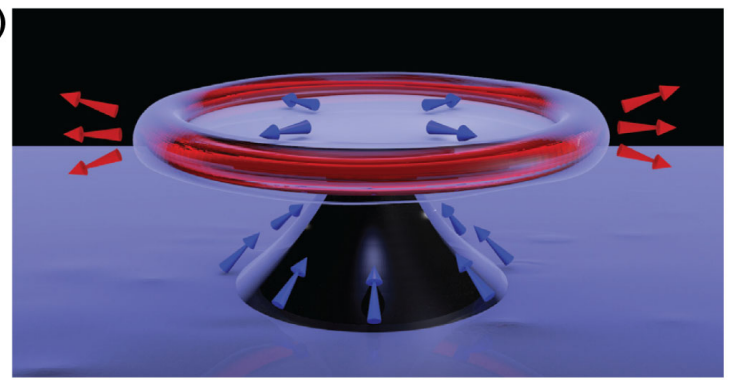

(c)

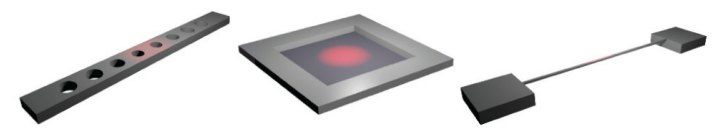

FIG. 1. (a) In bulk, a local heat source generates flow of superfluid helium (blue arrows) and counterflow of normal fluid (red arrow), imparting momentum onto the oscillator. (b) Representation of a microtoroid covered in a thin film of superfluid helium. Heat around the periphery caused by optical absorption generates fluid flow (blue arrows). At low pressures the superfluid then transitions directly into the gas phase and imparts momentum on evaporation (red arrows). (c) Superfluid-mediated photoconvective forcing may be readily extended to other optomechanical systems such as photonic-phononic crystal cavities, membranes, and nanostrings. Evaporation of a thin film from a photonic-phononic crystal cavity (left) due to localized optical heating would actuate the mechanical breathing modes commonly used in quantum optomechanics experiments. A mechanical membrane (middle) could be driven by bulk flow if one half was immersed in bulk superfluid helium via the mechanism of (a). The string modes of a nanostring (right) could be actuated by coating its top surface with an absorbing material upon which light is focused. This would cause preferential vertical evaporation of a thin superfluid film self-assembled on the device.

[33]. Furthermore, superfluid flow, once initiated, has been observed to persist for many hours without appreciable decay [34]. This unique flow persistence provides the possibility to apply persistent pressure on a mechanical element after the optical field is removed, in a form of nonvolatile optomechanical memory [17].

The configuration we use here to realize superfluid photoconvective forcing is represented in Fig. 1(b). A microtoroidal resonator is covered in a several-nanometerthick film of superfluid helium [35], which forms naturally due to van der Waals forces. Absorption of the circulating laser field at the microtoroid periphery (red glow) causes localized heating. This increase in temperature generates superfluid helium flow up the pedestal towards the heat source via the fountain effect (blue arrows). At the periphery, superfluid helium is evaporated (red arrows), resulting in a force on the microtoroid that, on average, is directed radially inwards. The magnitude of this radial force is given by

$$
\begin{aligned}
F_{\text {radial }} & =-\frac{d\left(m v_{\text {radial }}\right)}{d t} \\
& =\frac{4}{\pi^{2}} \dot{m} v_{\text {rms }}
\end{aligned}
$$

where $\dot{m}$ is the mass flow rate of evaporated helium. The net radial velocity $v_{\text {radial }}$ is calculated by integrating the contribution from isotropic evaporation in the outwards facing half-space with a root-mean-square (rms) velocity of

$$
v_{\mathrm{rms}}=\sqrt{\frac{3 k_{B} T_{\mathrm{evap}}}{m_{\mathrm{He}}}},
$$

where $T_{\text {evap }}$ is the temperature of evaporated atoms and $m_{\mathrm{He}}$ is the mass of a helium atom (see Supplemental Material [36]).

In steady state, the mass flow rate of the superfluid is determined by balancing the optical heat load with the energy dissipated through normal fluid counterflow or evaporation of the film (see Supplemental Material for further discussion [36]). While in bulk superfluid systems the energy dissipation is typically dominated by counterflow, for thin films the normal fluid fraction is viscously clamped to the surface [37], and evaporation dominates. To prevent the continuous accumulation of fluid at the heat source, the rate of evaporation must be equal to the influx from superfluid flow. For an absorbed optical power $P_{\text {abs }}$, the superfluid mass flow rate is then $\dot{m}=P_{\mathrm{abs}} /\left(L-\left\langle\mu_{\mathrm{vdW}}\right\rangle\right)$, where $L$ is the latent heat of vaporization and $\left\langle\mu_{\mathrm{vdW}}\right\rangle$ is the van der Waals potential of the superfluid film (see Supplemental Material [36]), and the resulting inward radial force from helium evaporation is

$$
F_{\text {radial }}=\frac{4}{\pi^{2}} \sqrt{\frac{3 k_{B} T_{\text {evap }}}{m_{\mathrm{He}}}} \frac{P_{\mathrm{abs}}}{L-\left\langle\mu_{\mathrm{vdW}}\right\rangle} .
$$

Note that, similar to photothermal forces [38], this expression is independent of the cavity finesse allowing photoconvective forces to be applied effectively where only a weak cavity, or no cavity, is present. For comparison, if the incident light is fully absorbed, the radiation pressure force is given by $F_{\mathrm{RP}}=P_{\text {abs }} \mathcal{F} / c$, where $\mathcal{F}$ is the cavity finesse and $c$ is the speed of light. For a $1 \mathrm{~K}$ superfluid evaporation temperature, the ratio $F_{\text {radial }} / F_{\mathrm{RP}} \sim 4 \times 10^{5} / \mathcal{F}$, indicating that, in our configuration, the superfluid photoconvective force is similar in magnitude to the radiation pressure force from a cavity with a finesse of around 400000 . For our 
experimental conditions, with a finesse of $\mathcal{F}=53000$, the superfluid force is predicted to be $\sim 1$ order of magnitude larger than the radiation pressure.

To experimentally realize this prediction, we use the setup shown in Fig. 2(a). A microtoroidal whisperinggallery-mode resonator [major radius $37.5 \mu \mathrm{m}$, minor radius $3.5 \mu \mathrm{m}$; Fig. 2(c)] is located inside the sample chamber of a helium-3 closed-cycle cryostat (Oxford Triton). Laser light at $1555.08 \mathrm{~nm}$ is coupled into a high-quality optical mode (linewidth $\kappa / 2 \pi=23.5 \mathrm{MHz}$ )
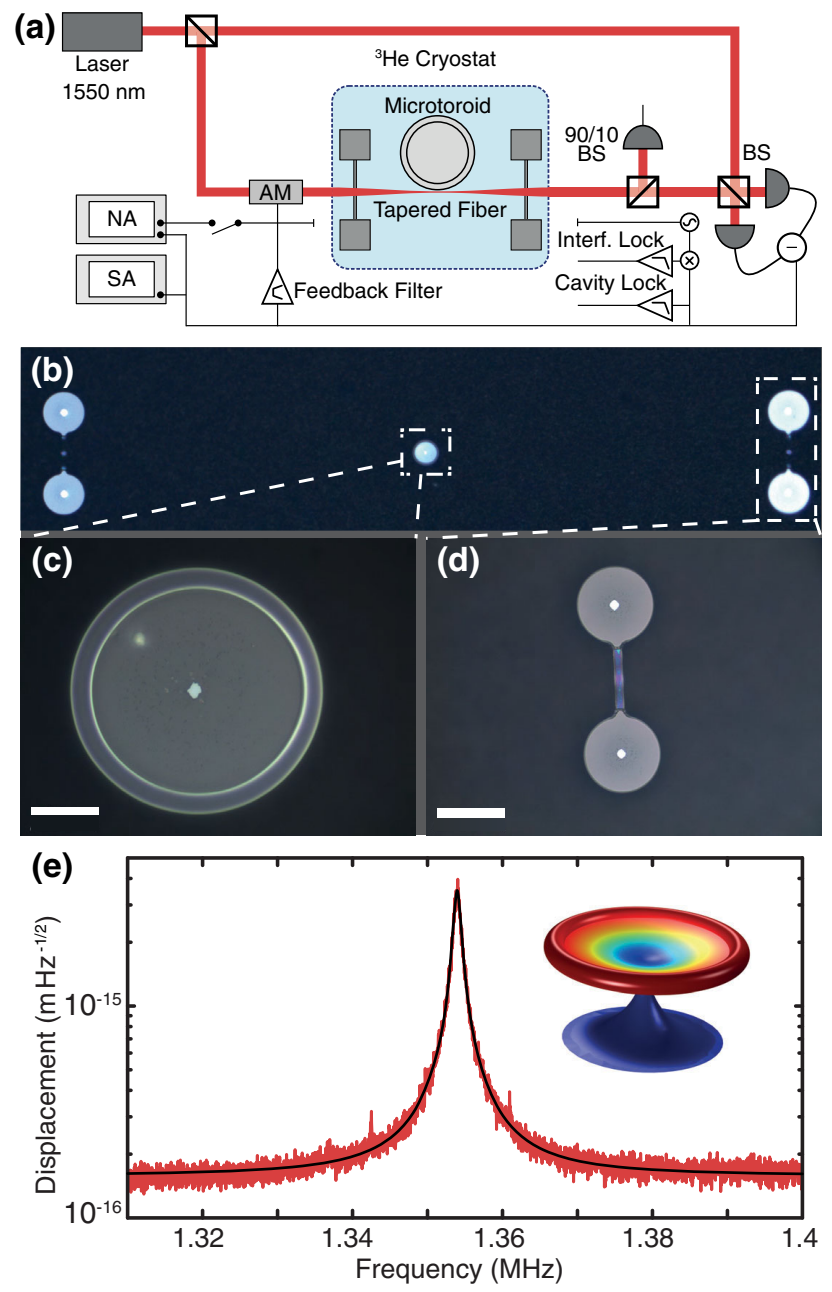

FIG. 2. (a) Experimental schematic. A microtoroid is nested inside an all-fiber interferometer and cooled by a He-3 refrigerator (BS, beam splitter; AM, amplitude modulator; SA, spectrum analyzer; NA, network analyzer). (b) Optical microscope image of the microtoroid used in these experiments, showing the support beams, one on either side, that are used to stabilize the tapered optical fiber. (c) Zoomed-in microscope image of the microtoroid. Scale bar is $20 \mu \mathrm{m}$ long. (d) Zoomed-in microscope image of a stabilization beam. The circular pads support a suspended beam that has been thinned to $200 \mathrm{~nm}$ thickness in order to minimize optical scattering loss. Scale bar is $100 \mu \mathrm{m}$ long. (e) Thermal motion of the flexural mode of a microtoroid at $3 \mathrm{~K}$. Inset: FEM simulation of the mechanical displacement profile. of the microtoroid via a tapered optical fiber. The tapered fiber rests on suspended stabilization beams fabricated near the microtoroid [Figs. 2(b) and 2(d)], ensuring that cryostat vibrations do not affect the taper-toroid separation. The microtoroid supports a number of intrinsic mechanical modes ranging in frequency from 1 to $50 \mathrm{MHz}$. The thermal motion of these modes is imprinted as phase fluctuations onto the optical field which are measured using homodyne detection. The radial forces applied by both radiation pressure and superfluid flow have optimal overlap with the radial breathing mode of the toroid, at $40 \mathrm{MHz}$. However, the superfluid forcing is observed to be ineffective above frequencies of approximately $2 \mathrm{MHz}$, possibly due to breakdown of superfluidity as the Landau critical velocity is reached [23]. Consequently, we perform experiments with the first-order flexural mode at $\Omega_{m} / 2 \pi=1.35 \mathrm{MHz}$, which has a mechanical dissipation rate of $\Gamma_{m} / 2 \pi=530 \mathrm{~Hz}$ at base temperature (559 $\mathrm{mK}$ ). The single-photon optomechanical coupling rate of this mode is measured as $g_{0} / 2 \pi=12.3 \mathrm{~Hz}$, and the Brownian fluctuations at $3 \mathrm{~K}$ are shown in Fig. 2(e) with the displacement profile from finite element modeling (FEM) shown in the inset.

To produce the superfluid film, the sample chamber is filled with low-pressure helium-4 gas (19 mbar at $2.9 \mathrm{~K})$ and cooled to base temperature. This gas pressure is specifically chosen to provide a superfluid film with a thickness such that the characteristic frequencies of third sound modes intrinsic to the superfluid film [35] do not overlap with the microtoroid mode. At $850 \mathrm{mK}$ the helium transitions directly from the gas phase to its superfluid state, forming a thin $(<5 \mathrm{~nm})$ superfluid layer over the chamber and its contents. To estimate the final temperature of the microtoroid, the flexural mode is monitored as the cryostat temperature is decreased from $10 \mathrm{~K}$ to $320 \mathrm{mK}$. Spectral analysis of the homodyne photocurrent gives the mechanical mode temperature via the integrated power spectral density. From $10 \mathrm{~K}$ to $600 \mathrm{mK}$ the microtoroid is well thermalized to the cryostat, as shown by the linear fit in Fig. 3(a); however, at lower cryostat temperatures the microtoroid mode temperature plateaus and is no longer in thermal equilibrium with the cryostat. We attribute this temperature deviation to the heat dissipated at the sample causing a thermal gradient between the microtoroid and cryostat cold plate.

To investigate the effects of optical absorption on the temperature of the microtoroid, we determine the integrated power spectral density of the mechanical mode as a function of laser power. The temperature is found to increase with increasing laser power, eventually causing a boiloff of the superfluid film, as shown in Fig. 3(b). As the laser power is increased over 2 orders of magnitude from $10 \mathrm{nW}$ to $2.1 \mu \mathrm{W}$, the mode temperature increases only modestly from 510 to $730 \mathrm{mK}$. Above $2.2 \mu \mathrm{W}$ the mode temperature jumps sharply to $3 \mathrm{~K}$, indicated by the 

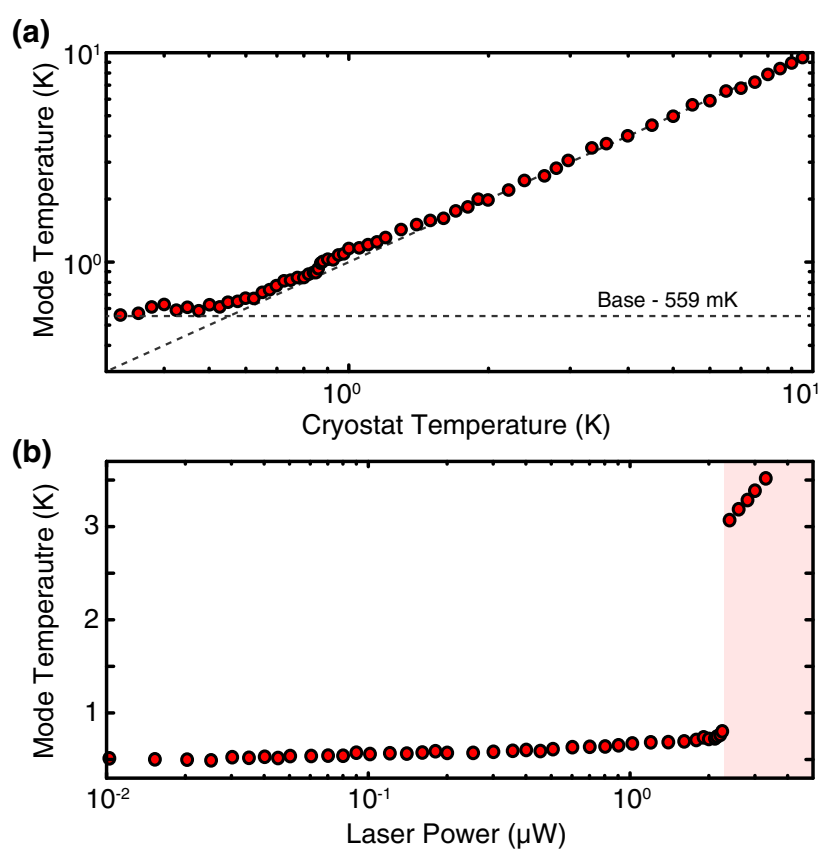

FIG. 3. (a) Mode temperature of the microtoroid flexural mode as the cryostat is cooled from 10 to $0.32 \mathrm{~K}$. The microtoroid reaches a base temperature of $0.56 \mathrm{~K}$ with $100 \mathrm{nW}$ of injected optical power. (b) Mode temperature of the flexural mode as the probe laser is increased from $10 \mathrm{nW}$ to $3.3 \mu \mathrm{W}$. Below $2.2 \mu \mathrm{W}$ the temperature increases slightly as the laser power is increased. Above $2.2 \mu \mathrm{W}$ the superfluid boils off, causing a sharp rise in mode temperature.

red shaded region in Fig. 3(b). This threshold behavior manifests due to Landau's critical velocity, which sets an upper limit on the superfluid flow rate (see Supplemental Material [36]). This results in a thermal runaway process, wherein the superfluid can no longer be replenished at the periphery of the microtoroid as fast as it evaporates and therefore boils off completely. The microtoroid is then no longer effectively thermally anchored to the cryostat, and the final mode temperature is dominated by laser heating.

To investigate the optical forces present in the system, a constant optical amplitude modulation is applied at the frequency of the flexural mode as the cryostat temperature is varied (Fig. 4). This applies resonant forces on the mode, through both radiation pressure and, below the superfluid transition temperature, superfluid flow. The mechanical response to this drive is measured via homodyne detection of the phase quadrature of the output field. At temperatures above the superfluid transition, the optical force originates from radiation pressure alone and is essentially independent of temperature (see right-hand inset of Fig. 4). However, upon formation of a superfluid layer, indicated by the blue shaded region in Fig. 4, the response of the flexural mode to the laser drive abruptly increases by $21 \mathrm{~dB}$ to a maximum of $540 \mathrm{fN}$ (left-hand inset of Fig. 4). Taking into account the poor overlap between the flexural mode and the radial evaporative force $(0.037 \%$ calculated from FEM) gives a

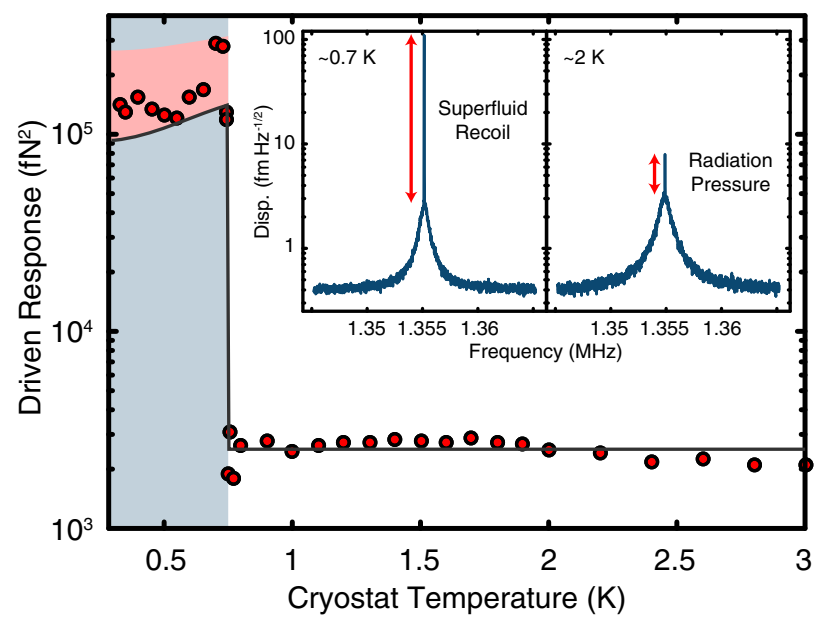

FIG. 4. Driven response of the flexural mode as the cryostat is cooled (red points), showing a step increase in response at the superfluid transition temperature. Black line represents a theoretical fit to the data. The gray shaded area indicates that a superfluid layer has formed on the microtoroid surface. The pink shaded area represents the theoretical force if $T_{\text {evap }}$ is up to $1 \mathrm{~K}$ higher than the mode temperature. Inset: Displacement spectrum of the flexural mode at 0.7 and $2 \mathrm{~K}$ with a coherent drive applied via optical amplitude modulation. The response to coherent drive is shown to increase with the presence of superfluid helium.

total superfluid photoconvective force of $1.46 \mathrm{nN}$. This increased response in the presence of the superfluid is in good agreement with theoretical predictions, corresponding to a superfluid photoconvective force that is a factor of 11 larger than the radiation pressure. The measured superfluid convective force decreases in magnitude as the temperature is reduced away from the transition temperature (see Fig. 4). This occurs because of the reduced rms velocity of the evaporated atoms [see Eq. (3)], with colder atoms contributing less recoil to the microtoroid. This behavior is accurately predicted by our model, where the superfluid evaporation temperature in Eq. (4) is equated to the measured microtoroid mode temperature $T_{m}$. However, the observed superfluid forces are found consistently to be larger than predicted by the model, with a maximum deviation of approximately $60 \%$. We attribute this discrepancy to a temperature differential existing between the evaporated atoms and the helium film. It has been shown that helium atoms evaporated from a superfluid thin film have a temperature that is up to $1 \mathrm{~K}$ hotter than the film temperature, dependent on the total heat applied to the liquid $[39,40]$. To account for this phenomenon, we include in Fig. 4 a theoretical band (pink shading) showing the expected applied force for atoms that are evaporated with temperatures $T_{\text {evap }}$ ranging from the mode temperature $T_{m}$ to $T_{m}+1 \mathrm{~K}$.

As a specific example of an application that takes advantage of the enhanced optical force provided by the superfluid, we perform feedback cooling on the microtoroid mode. This is done by passing the homodyne 


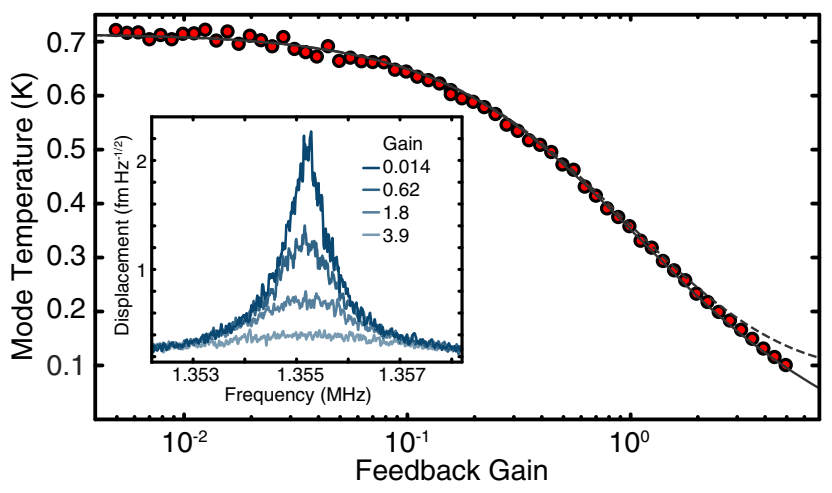

FIG. 5. Feedback cooling of the flexural mode from 715 to $137 \mathrm{mK}$ using superfluid-mediated photoconvective forcing. With a fixed probe power of $1.9 \mu \mathrm{W}$, the feedback gain is varied over 3 orders of magnitude, showing good agreement with the estimated mode temperature from in-loop measurements (solid line). The out-of-loop mode temperature is then inferred by a transformation (dashed line) that is derived in the Supplemental Material [36]. Inset: Displacement spectrum of the flexural mode with varying feedback gain.

photocurrent through various filter and amplification stages, then feeding it into an amplitude modulator placed before the microtoroid [see Fig. 2(a)]. Provided that the phase of the feedback loop has been chosen correctly to provide a force that opposes the velocity of the mode, the thermal motion of the 1.35-MHz flexural mode is reduced via cold damping [41]. Figure 5 shows that as the feedback gain is increased, the microtoroid mode temperature decreases, in excellent agreement with theory [42]. The flexural mode is thus cooled from 715 to $137 \mathrm{mK}$, with a final mechanical occupancy of $n=2110 \pm 40$ phonons.

In general for feedback cooling, the final mechanical occupancy has contributions both from the optomechanical coupling rate, which defines the conditional variance of the oscillator following measurement, and by the maximum strength of the feedback force (see Supplemental Material, Sec. VI.2 [36] and Ref. [43]). In our case, it is the relatively low optomechanical coupling rate of the microtoroid flexural mode that limits $n$. We estimate that, for our mechanical mode and cryostat temperature and with the maximum feedback force magnitude of $1.46 \mathrm{nN}$ we achieve here, the superfluid feedback force would only become a limiting factor for phonon numbers below $n \approx 0.015$ (see Supplemental Material, Sec. VI.2 [36]). Therefore, with improved microtoroid parameters, such as those achieved in Refs. [13,44], ground state cooling should be possible using superfluid forces. Nonclassical mechanical states could also be generated by combining superfluid feedback forces with backaction evading [26-28] or nonlinear measurements [29,30].

While we have already demonstrated an order of magnitude improvement in optical force over radiation pressure using evaporative recoil in thin superfluid films, for completeness we discuss in the Supplemental Material [36] the magnitude of the forces arising from superflow and normal fluid counterflow during heat transport in bulk superfluid [as illustrated in Fig. 1(a)]. By choosing the right experimental parameters, we show that the force could be further increased by over an order of magnitude compared to the thin film case, enabling optical forces more than 2 orders of magnitude larger than what is achievable with radiation pressure even in a high-finesse cavity.

The strongest known optical actuation capabilities are provided by the photothermal interaction [31]. Indeed, the first optomechanical system to demonstrate cooling via dynamical backaction was based on this mechanism [38]. Further, it has been shown that photothermal forces should enable optomechanical cooling to the ground state, without requiring sideband resolution [31], thus enabling efficient cooling of low-frequency mechanical oscillators. However, accessing large photothermal forces requires strong optical absorption and a large thermal expansion coefficient, limiting devices to specific materials and geometries, and additionally precludes cryogenic operation as the thermal expansion coefficient of most materials reduces by several orders of magnitude when cooled to cryogenic temperatures. Furthermore, the characteristic bandwidth is defined by the typically slow thermalization rate of the device material. Superfluid convective and evaporative forces could alleviate these constraints, allowing new regimes to be realized characterized by fast, strong actuation, with the potential to incorporate superfluid-enhanced optical forcing into existing cryogenic optomechanical systems. Strong actuation can, alternatively, be achieved electrically, for example, using electrostatic [42] or piezoelectric [45] forces. While these techniques offer considerable advantages in terms of their configurability and scalability, they require integration of electrical circuitry into the optomechanical device-an area that, we note, has seen rapid recent progress [46-48]. Piezoelectric actuation is, further, possible only when using a restricted class of materials.

Superfluid convective and evaporative forces may find applications where strong optical forces are required in the absence of an optical cavity, for example, in photonic circuits $[1,2,49]$ or cryogenic MEMS [50]. It should also be possible to design systems where the heat source is applied at a location spatially remote from the resultant force, and in a range of geometries (see Fig. 6) to apply not only forces, but also torques [Fig. 6(c)], at the microscale. Furthermore, it may be possible to design architectures that benefit from the persistent flow that is uniquely available in superfluids. Remarkably, flows in superfluid helium- 4 have been measured to persist for times exceeding 6 hours, without any observed decay [34]. By engineering chirality in the structure of an optical microcavity [see Fig. 6(f)], this persistent flow could be converted into a persistent circulation, exerting a radial pressure on the cavity and thereby introducing a static shift to its resonance frequencies. Our modeling indicates that shifts larger than the cavity linewidth can be achieved with current whispering-gallery-mode devices, including 
(a)

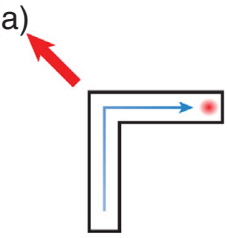

(b)

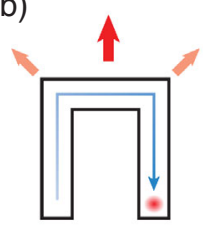

(d)

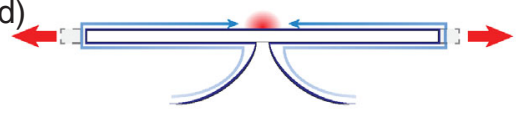

(e)

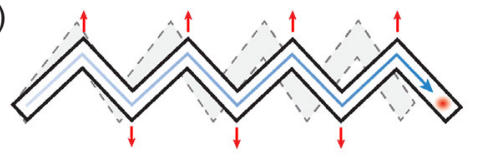

(c)

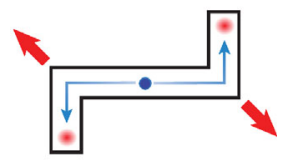

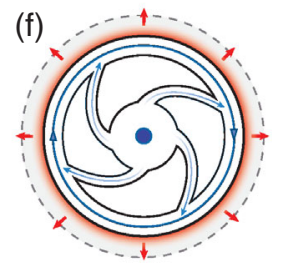

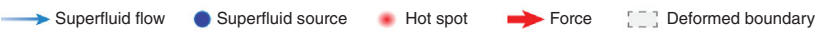

FIG. 6. By varying the geometry, superfluid flow could be used to apply a large range of different forces including (a), (b) linear force, (c) torque, (d)-(f) examples of geometries designed to efficiently leverage forces from superfluid flow. (d) Expansive force that is efficiently coupled to a microdisk or microtoroid, (e) compressive force, and (f) long-lived centrifugal forces resulting from persistent current flow.

both microtoroids and microdisks (see Supplemental Material, Sec. IV [36]). This could, for example, be used for nonvolatile switchable optical routing or even a nonvolatile optomechanical memory [17].

In conclusion, we demonstrate photoconvective forcing of a mechanical oscillator based on superfluid flow and recoil. This enables large optical forces at cryogenic temperatures, in contrast to photothermal forcing mechanisms that are generally precluded from cryogenic operation. The exceptionally high thermal conductivity of superfluid helium provides good thermal anchoring to the environment and permits the application of fast optical forces, while the persistence of superfluid flow and the ability to apply forces remotely provide the prospect for new applications. The self-assembling nature of superfluid helium means this technique may be relatively straightforwardly incorporated into other cryogenic optomechanical systems.

This research was funded by the Australian Research Council Centre of Excellence CE110001013. W. P. B. is supported by the Australian Research Council Future Fellowship No. FT140100650. Device fabrication was undertaken within the Queensland Node of the Australian Nanofabrication Facility.

D. L. M. and G. I. H. contributed equally to this work.

[1] M. Li, W. H. P. Pernice, C. Xiong, T. Baehr-Jones, M. Hochberg, and H. X. Tang, Harnessing Optical Forces in Integrated Photonic Circuits, Nature (London) 456, 480 (2008).
[2] J. Roels, I. De Vlaminck, L. Lagae, B. Maes, D. Van Thourhout, and R. Baets, Tunable Optical Forces between Nanophotonic Waveguides, Nat. Nanotechnol. 4, 510 (2009).

[3] A. Ashkin and J.M. Dziedzic, Optical Trapping and Manipulation of Viruses and Bacteria, Science 235, 1517 (1987).

[4] M. P. MacDonald, G. C. Spalding, and K. Dholakia, Microfluidic Sorting in an Optical Lattice, Nature (London) 426, 421 (2003).

[5] T. P. Burg, M. Godin, S. M. Knudsen, W. Shen, G. Carlson, J. S. Foster, K. Babcock, and S. R. Manalis, Weighing of Biomolecules, Single Cells and Single Nanoparticles in Fluid, Nature (London) 446, 1066 (2007).

[6] M. A. Taylor, J. Janousek, V. Daria, J. Knittel, B. Hage, H. A. Bachor, and W. P. Bowen, Biological Measurement beyond the Quantum Limit, Nat. Photonics 7, 229 (2013).

[7] H. J. Mamin and D. Rugar, Sub-Attonewton Force Detection at Millikelvin Temperatures, Appl. Phys. Lett. 79, 3358 (2001).

[8] S. Forstner, S. Prams, J. Knittel, E. D. van Ooijen, J. D. Swaim, G. I. Harris, A. Szorkovszky, W. P. Bowen, and H. Rubinsztein-Dunlop, Cavity Optomechanical Magnetometer, Phys. Rev. Lett. 108, 120801 (2012).

[9] J. Chaste, A. Eichler, J. Moser, G. Ceballos, R. Rurali, and A. Bachtold, A Nanomechanical Mass Sensor with Yoctogram Resolution, Nat. Nanotechnol. 7, 301 (2012).

[10] J. Beugnon, C. Tuchendler, H. Marion, A. Gaetan, Y. Miroshnychenko, Y. R. P. Sortais, A. M. Lance, M. P. A. Jones, G. Messin, A. Browaeys, and P. Grangier, TwoDimensional Transport and Transfer of a Single Atomic Qubit in Optical Tweezers, Nat. Phys. 3, 696 (2007).

[11] C. Orzel, A. K. Tuchman, M. L. Fenselau, M. Yasuda, and M. A. Kasevich, Squeezed States in a Bose-Einstein Condensate, Science 291, 2386 (2001).

[12] M. Greiner, O. Mandel, T. Esslinger, T. W. Hansch, and I. Bloch, Quantum Phase Transition from a Superfluid to a Mott Insulator in a Gas of Ultracold Atoms, Nature (London) 415, 39 (2002).

[13] E. Verhagen, S. Deleglise, S. Weis, A. Schliesser, and T. J. Kippenberg, Quantum-Coherent Coupling of a Mechanical Oscillator to an Optical Cavity Mode, Nature (London) 482, 63 (2012).

[14] D. W. C. Brooks, T. Botter, S. Schreppler, T. P. Purdy, N. Brahms, and D.M. Stamper-Kurn, Non-Classical Light Generated by Quantum-Noise-Driven Cavity Optomechanics, Nature (London) 488, 476 (2012).

[15] J. Chan, T. P. M. Alegre, A. H. Safavi-Naeini, J. T. Hill, A. Krause, S. Groblacher, M. Aspelmeyer, and O. Painter, Laser Cooling of a Nanomechanical Oscillator into Its Quantum Ground State, Nature (London) 478, 89 (2011).

[16] J. Rosenberg, Q. Lin, and O. Painter, Static and Dynamic Wavelength Routing via the Gradient Optical Force, Nat. Photonics 3, 478 (2009).

[17] M. Bagheri, M. Poot, M. Li, W. P. H. Pernice, and H.X. Tang, Dynamic Manipulation of Nanomechanical Resonators in the High-Amplitude Regime and Non-Volatile Mechanical Memory Operation, Nat. Nanotechnol. 6, 726 (2011). 
[18] K. Usami, A. Naesby, T. Bagci, B. M. Nielsen, J. Liu, S. Stobbe, P. Lodahl, and E. S. Polzik, Optical Cavity Cooling of Mechanical Modes of a Semiconductor Nanomembrane, Nat. Phys. 8, 168 (2012).

[19] R. A. Barton, I. R. Storch, V. P. Adiga, R. Sakakibara, B. R. Cipriany, B. Ilic, S. P. Wang, P. Ong, P. L. McEuen, J. M. Parpia, and H. G. Craighead, Photothermal Self-Oscillation and Laser Cooling of Graphene Optomechanical Systems, Nano Lett. 12, 4681 (2012).

[20] S. W. Stahl, E. M. Puchner, and H. E. Gaub, Photothermal Cantilever Actuation for Fast Single-Molecule Force Spectroscopy, Rev. Sci. Instrum. 80, 073702 (2009).

[21] F. Marino and F. Marin, Chaotically Spiking Attractors in Suspended-Mirror Optical Cavities, Phys. Rev. E 83, 015202 (2011).

[22] J. F. Allen and H. Jones, New Phenomena Connected with Heat Flow in Helium II, Nature (London) 141, 243 (1938).

[23] L. Landau, Theory of Superfluidity of Helium II, Phys. Rev. 60, 356 (1941).

[24] L. Tisza, Transport Phenomena in Helium II, Nature (London) 141, 913 (1938).

[25] D. J. Wilson, V. Sudhir, N. Piro, R. Schilling, A. Ghadimi, and T. J. Kippenberg, Measurement-Based Control of a Mechanical Oscillator at Its Thermal Decoherence Rate, Nature (London) 524, 325 (2015).

[26] V. B. Braginsky, Y. I. Vorontsov, and K. S. Thorne, Quantum Nondemolition Measurements, Science 209, 547 (1980).

[27] E. E. Wollman, C. U. Lei, A. J. Weinstein, J. Suh, A. Kronwald, F. Marquardt, A. A. Clerk, and K. C. Schwab, Quantum Squeezing of Motion in a Mechanical Resonator, Science 349, 952 (2015).

[28] A. Szorkovszky, A. C. Doherty, G. I. Harris, and W. P. Bowen, Mechanical Squeezing via Parametric Amplification and Weak Measurement, Phys. Rev. Lett. 107, 213603 (2011).

[29] J. D. Thompson, B. M. Zwickl, A. M. Jayich, F. Marquardt, S. M. Girvin, and J. G. E. Harris, Strong Dispersive Coupling of a High-Finesse Cavity to a Micromechanical Membrane, Nature (London) 452, 72 (2008).

[30] G. A. Brawley, M. R. Vanner, P. E. Larsen, S. Schmid, A. Boisen, and W.P. Bowen, Non-Linear Optomechanical Measurement of Mechanical Motion, Nat. Commun. 7, 10988 (2016).

[31] J. Restrepo, J. Gabelli, C. Ciuti, and I. Favero, Classical and Quantum Theory of Photothermal Cavity Cooling of a Mechanical Oscillator, C.R. Phys. 12, 860 (2011).

[32] S. M. Meenehan, J. D. Cohen, G. S. MacCabe, F. Marsili, M. D. Shaw, and O. Painter, Pulsed Excitation Dynamics of an Optomechanical Crystal Resonator near Its Quantum Ground State of Motion, Phys. Rev. X 5, 041002 (2015).

[33] M. Wu, A. C. Hryciw, C. Healey, D. P. Lake, H. Jayakumar, M. R. Freeman, J. P. Davis, and P. E. Barclay, Dissipative and Dispersive Optomechanics in a Nanocavity Torque Sensor, Phys. Rev. X 4, 021052 (2014).
[34] I. Rudnick, H. Kojima, W. Veith, and R. S. Kagiwada, Observation of Superfluid-Helium Persistent Current by Doppler-Shifted Splitting of Fourth-Sound Resonance, Phys. Rev. Lett. 23, 1220 (1969).

[35] G. I. Harris, D. L. McAuslan, E. Sheridan, Y. Sachkou, C. Baker, and W.P. Bowen, Laser Cooling and Control of Excitations in Superfluid Helium, Nat. Phys., doi:10.1038/nphys3714 (2016).

[36] See Supplemental Material at http://link.aps.org/ supplemental/10.1103/PhysRevX.6.021012 for a detailed discussion of photoconvective forces in thin films and bulk, as well as feedback cooling principles.

[37] K. R. Atkins, Third and Fourth Sound in Liquid Helium II, Phys. Rev. 113, 962 (1959).

[38] C. H. Metzger and K. Karrai, Cavity Cooling of a Microlever, Nature (London) 432, 1002 (2004).

[39] D. S. Hyman, M. O. Scully, and A. Widom, Evaporation from Superfluid Helium, Phys. Rev. 186, 231 (1969).

[40] K. Andres, R. C. Dynes, and V. Narayanamurti, Velocity Spectrum of Atoms Evaporating from a Liquid He Surface at Low Temperatures, Phys. Rev. A 8, 2501 (1973).

[41] P. F. Cohadon, A. Heidmann, and M. Pinard, Cooling of a Mirror by Radiation Pressure, Phys. Rev. Lett. 83, 3174 (1999).

[42] K. H. Lee, T. G. McRae, G. I. Harris, J. Knittel, and W. P. Bowen, Cooling and Control of a Cavity Optoelectromechanical System, Phys. Rev. Lett. 104, 123604 (2010).

[43] W. P. Bowen and G. J. Milburn, Quantum Optomechanics (Springer, London, 2015).

[44] G. Anetsberger, R. Rivire, A. Schliesser, O. Arcizet, and T. J. Kippenberg, Ultralow-Dissipation Optomechanical Resonators on a Chip, Nat. Photonics 2, 627 (2008).

[45] C. Xiong, L. Fan, X. Sun, and H.X. Tang, Cavity Piezooptomechanics: Piezoelectrically Excited, Optically Transduced Optomechanical Resonators, Appl. Phys. Lett. 102, 021110 (2013).

[46] J. Bochmann, A. Vainsencher, D. D. Awschalom, and A. N. Cleland, Nanomechanical Coupling between Microwave and Optical Photons, Nat. Phys. 9, 712 (2013).

[47] T. Bagci, A. Simonsen, S. Schmid, L. G. Villanueva, E. Zeuthen, J. Appel, J. M. Taylor, A. Srensen, K. Usami, A. Schliesser, and E. S. Polzik, Optical Detection of Radio Waves through a Nanomechanical Transducer, Nature (London) 507, 81 (2014).

[48] R. W. Andrews, R. W. Peterson, T. P. Purdy, K. Cicak, R. W. Simmonds, C. A. Regal, and K. W. Lehnert, Bidirectional and Efficient Conversion between Microwave and Optical Light, Nat. Phys. 10, 321 (2014).

[49] M. Li, W. H. P. Pernice, and H. X. Tang, Tunable Bipolar Optical Interactions between Guided Lightwaves, Nat. Photonics 3, 464 (2009).

[50] S. Waldis, F. Zamkotsian, P.-A. Clerc, W. Noell, M. Zickar, and N. de Rooij, Arrays of High Tilt-Angle Micromirrors for Multiobject Spectroscopy, IEEE J. Sel. Top. Quantum Electron. 13, 168 (2007). 